\title{
The potential of dietary polyunsaturated fatty acids to modulate eicosanoid synthesis and reproduction in Daphnia magna: A gene expression approach
}

\author{
Nina Schlotz ${ }^{\text {a,* }}$, Jesper Givskov Sørensen ${ }^{\text {b }}$, Dominik Martin-Creuzburg a \\ a Limnological Institute, University of Konstanz, Mainaustrasse 252, 78464 Konstanz, Germany \\ b Department of Bioscience, Aarhus University, Vejlsøvej 25, 8600 Silkeborg, Denmark
}

Keywords:

Arachidonic acid

Daphnia magna

Eicosanoids

Eicosapentaenoic acid

Food quality

Gene expression

Nutrition

Vitellogenin

\begin{abstract}
A B S T R A C T
Nutritional ecology of the aquatic model genus Daphnia has received much attention in past years in particular with regard to dietary polyunsaturated fatty acids (PUFAs) which are crucial for growth and reproduction. Besides their significant role as membrane components, C20 PUFAs serve as precursors for eicosanoids, hormone-like mediators of reproduction, immunity and ion transport physiology. In the present study we investigate transcriptomic changes in Daphnia magna in response to different algal food organisms substantially differing in their PUFA composition using quantitative real-time PCR and relate them to concomitantly documented life history data. The selection of target genes includes representatives that have previously been shown to be responsive to the eicosanoid biosynthesis inhibitor ibuprofen. The beneficial effect of C20 PUFA-rich food on reproduction and population growth rates was accompanied by an increased vitellogenin (DmagVtg1) gene expression in D. magna. Additionally, genes involved in eicosanoid signaling were particularly influenced by dietary C20 PUFA availability. For example, the cyclooxygenase gene (Cox), coding for a central enzyme in the eicosanoid pathway, was highly responsive to the food treatments. Our results suggest that dietary PUFAs are fundamental in D. magna physiology as substrate for eicosanoid synthesis and that these eicosanoids are important for $D$. magna reproduction.
\end{abstract}

\section{Introduction}

Species of the genus Daphnia have become important model organisms in ecology, ecotoxicology and evolutionary biology (Ebert, 2005; Lampert, 2011). As keystone herbivores in freshwater food webs they provide a crucial link between primary and secondary production. Owing to the long history of ecological research, our knowledge of e.g. life history traits or phenotypic plasticity is vast compared to many other model organisms. With the fully sequenced genome of Daphnia pulex another major advantage was given to Daphnia as a model (Colbourne et al., 2011; Ebert, 2011). However, our understanding of the physiological mechanisms involved in coping with environmental stress situations (e.g. temperature changes, predation, pathogen challenge, nutrient limitations) is still scarce.

Abbreviations: ALA, $\alpha$-linolenic acid; ARA, arachidonic acid; CLECT, C-type lectin like; COX, cyclooxygenase; DGLA, dihomo- $\gamma$-linolenic acid; DHA, docosahexaenoic acid; DmagVTG1, vitellogenin1; EPA, eicosapentaenoic acid; FABP3, fatty acid binding protein 3; GLA, $\gamma$-linolenic acid; GPX, glutathione peroxidase; JHE, juvenile hormone esterase; LIN, linoleic acid; LIP, triacylglycerol lipase; LOX, lipoxygenase; LTB4DH, leukotriene B4 12-hydroxydehydrogenase; MUFA, monounsaturated fatty acid; PUFA, polyunsaturated fatty acid; SAFA, saturated fatty acid; SDA, stearidonic acid.

* Corresponding author. Tel.: + 497531 882930; fax: + 497531883533.

E-mail addresses: Nina.Schlotz@uni-konstanz.de (N. Schlotz), jgs@dmu.dk (J.G. Sørensen), Dominik.Martin-Creuzburg@uni-konstanz.de (D. Martin-Creuzburg).
One of the factors greatly influencing Daphnia's performance is nutrition. Besides the obvious need to obtain enough food, i.e. the role of food quantity (Pietrzak et al., 2010), it also proved to be crucial to consume food of good quality in order to meet the requirements for optimal growth and reproduction. Even food organisms that are nontoxic and readily assimilated can be of poor quality (Lampert, 1977), one reason being the lack of essential elemental or biochemical nutrients, which impairs the fitness of the consumer. Among the elements, phosphorus $(\mathrm{P})$ and nitrogen $(\mathrm{N})$ are by far the best-studied representatives (Sterner and Elser, 2002). Among biochemical food quality constraints are, for example, essential lipids and amino acids. Amino acids have been reported recently to affect population growth and the reproductive mode of daphnids (Koch et al., 2011). As yet, however, the majority of studies on biochemical food quality constraints have focused on polyunsaturated fatty acids (PUFAs) and sterols (Müller-Navarra et al., 2000; von Elert, 2002; Martin-Creuzburg et al., 2009). The main sterol in animals is cholesterol. It is an integral part of membranes where it influences fluidity and permeability and thus plays a role in temperature acclimation (Mouritsen and Zuckermann, 2004). Furthermore, in arthropods, it serves as precursor for moulting hormones, the ecdysteroids (Mykles, 2011). The lack of sterols has been proposed to be a major constraint of energy transfer at the Daphnia-cyanobacteria interface (Martin-Creuzburg et al., 2008) and has shown to be one reason for the poor food quality of heterotrophic 
bacteria (Martin-Creuzburg et al., 2011). The role of PUFAs has been investigated using different approaches. Correlative field studies suggested limitations by different PUFAs in the field (Müller-Navarra, 1995; Müller-Navarra et al., 2000; Wacker and Von Elert, 2001) and results from laboratory growth experiments corroborate the importance of PUFAs for Daphnia fitness by demonstrating for example a better performance under temperature stress and revealing allocation patterns during PUFA deficiencies (Wacker and Martin-Creuzburg, 2007; Masclaux et al., 2009; Martin-Creuzburg et al., 2012). However, there is a lack of knowledge concerning the mechanisms underlying these beneficial effects as most of the available research merely compares various fitness parameters as a function of the dietary PUFA content.

PUFAs not only are indispensable components of membranes, where they alter membrane properties and contribute to, e.g., temperature acclimation, three of them also are precursors of a family of bioactive molecules, the eicosanoids (Stanley, 2000; Guschina and Harwood, 2006; van Meer et al., 2008). These three precursor PUFAs are dihomo- $\gamma$-linolenic acid (DGLA, 20:3n-6), arachidonic acid (ARA, 20:4n-6), and eicosapentaenoic acid (EPA, 20:5n-3). They can be metabolized by a cascade of enzymes to prostaglandins, leukotrienes, and thromboxanes. In mammals, there are three distinct pathways leading to formation of eicosanoids (Stanley, 2000); bioinformatics data suggests that only two of these pathways are present in Daphnia (Heckmann et al., 2008b). The genomic structure of the involved pathway ("arachidonic acid pathway", termed after the main substrate ARA) reveals significant expansion and contraction of relevant gene families in Pancrustacea and the lineage leading to $D$. pulex (Colbourne et al., 2011). This gain or loss of genes indicates evolutionary recent and extensive restructuring of an important metabolic pathway.

Even though there are a considerable number of studies that argue for the importance of PUFAs in invertebrate physiology, few are able to conclusively attribute the observed effects to actions exerted by these essential lipids or their metabolites. In this first attempt to elucidate mechanisms underlying the beneficial effects of dietary lipids on Daphnia growth and reproduction, we adopted and modified the idea of Heckmann et al. (2008a) and related life history parameters to gene expression. Yet, instead of applying a substance interfering with the pathway of interest we provided food organisms of different lipid quality. Heckmann et al. (2008a) used ibuprofen, one of the best-known non-steroidal anti-inflammatory drugs, to find out if the mode of action of this eicosanoid biosynthesis inhibitor in D. magna is akin to that in mammals. In mammals, ibuprofen works by inhibiting the enzyme cyclooxygenase (COX) which comprises, together with lipoxygenase (LOX), the central part of the eicosanoid synthesis pathway. The genes examined here are involved in lipid metabolism and the immune and/or endocrine system, all of which might be affected by the availability of dietary PUFAs; furthermore, most of them were responsive to ibuprofen in the study by Heckmann et al. (2008a). By simultaneously investigating life history traits and gene expression responses of $D$. magna we hope to gain first insights in how important pathways are influenced by PUFAmediated food quality and to reveal new avenues that should be explored in future studies.

\section{Material and methods}

\subsection{Food organisms}

We chose three algae differing in their lipid profiles for the growth experiments: the green alga Scenedesmus obliquus (SAG 276-3a), the eustigmatophyte Nannochloropsis limnetica (SAG 18.99), and the cryptophyte Cryptomonas sp. (SAG 26.80). Algae were obtained from the culture collection of the University of Göttingen (SAG, Germany).

The algae were each cultured semi-continuously in modified Woods Hole (WC) medium (Guillard, 1975) in aerated $5 \mathrm{~L}$ vessels $\left(20^{\circ} \mathrm{C}\right.$; dilution rate: $0.2 \mathrm{~d}^{-1}$; illumination: $100 \mathrm{mmol}$ quanta $\mathrm{m}^{-2} \mathrm{~s}^{-1}$ ). Food suspensions were produced by centrifugation of the harvested algae and resuspension in fresh medium. Carbon concentrations were estimated from photometric light extinctions $(480 \mathrm{~nm})$ and from previously determined carbon-extinction equations. The carbon-light extinction regressions were confirmed by subsequent carbon analysis of the food suspensions.

\subsubsection{Biochemical analyses}

Fatty acids - for the analysis of fatty acids approximately $1 \mathrm{mg}$ particulate organic carbon (POC) was filtered separately onto precombusted GF/F filters (Whatman, $25 \mathrm{~mm}$ ). Total lipids were extracted three times from filters with dichloromethane/methanol $(2: 1, v / v)$. Pooled cell-free extracts were evaporated to dryness under a nitrogen stream. The lipid extracts were transesterified with $3 \mathrm{M}$ methanolic $\mathrm{HCl}\left(60^{\circ} \mathrm{C}, 20 \mathrm{~min}\right)$. Subsequently, fatty acid methyl esters (FAMEs) were extracted three times with $2 \mathrm{~mL}$ of iso-hexane. The lipid-containing fraction was evaporated to dryness under nitrogen and resuspended in a volume of $20 \mu \mathrm{L}$ iso-hexane. Lipids were analyzed by gas chromatography on a HP 6890 GC equipped with a flame ionization detector (FID) and a DB-225 (J\&W Scientific, $30 \mathrm{~m} \times 0.25 \mathrm{~mm}$ ID $\times 0.25 \mathrm{~mm}$ film) capillary column to analyze FAMEs. Details of GC configurations for the analysis of FAMEs are given elsewhere (Martin-Creuzburg et al., 2010). FAMEs were quantified by comparison with the internal standard (C23:0 ME) of known concentration, considering response factors determined previously with lipid standards (Sigma-Aldrich). FAMEs were identified by their retention times and their mass spectra, which were recorded with a gas chromatograph-mass spectrometer (Agilent Technologies, $5975 C$ ) equipped with a fused-silica capillary column (DB-225MS, $\mathrm{J} \& \mathrm{~W}$ ). Spectra were recorded between 50 and $600 \mathrm{Da}$ in the electron impact ionization mode. The limit for quantitation of fatty acids was $20 \mathrm{ng}$. The absolute amount of each lipid was related to the POC.

Elemental composition - aliquots of food suspensions were filtered onto precombusted glass fiber filters (Whatman GF/F, $25 \mathrm{~mm}$ diameter) and analyzed for POC and nitrogen using an EuroEA3000 elemental analyzer (HEKAtech $\mathrm{GmbH}$, Wegberg, Germany). For the determination of particulate phosphorus, aliquots were collected on acid-rinsed polysulfone filters (HT-200; Pall, Ann Arbor, MI, USA) and digested with a solution of $10 \%$ potassium peroxodisulfate and $1.5 \%$ sodium hydroxide for $60 \mathrm{~min}$ at $121^{\circ} \mathrm{C}$. Soluble reactive phosphorus was determined using the molybdate-ascorbic acid method (Greenberg et al., 1985).

\subsection{Experimental design}

For all experiments the same clone of $D$. magna $\left(\mathrm{HO}_{2}\right.$, originally isolated in Hungary) was used. Stock cultures were cultivated in artificial medium (Aachener Daphnien Medium (ADaM)), modified after Klüttgen et al. (1994) and fed with saturating amounts of S. obliquus. All experiments were conducted with third-clutch neonates born within $12 \mathrm{~h}$ at $20^{\circ} \mathrm{C}$.

\subsubsection{Life history experiment}

Animals were kept individually in $80 \mathrm{~mL}$ ADaM. They were randomly assigned to one of the three food regimes: S. obliquus (Scen), N. limnetica (Nanno), and Cryptomonas sp. (Crypto). Every other day, they were transferred to fresh medium and received algal food suspensions corresponding to $3 \mathrm{mg}$ carbon per litre. During the experiment mortality and reproduction were recorded.

Population growth rates were estimated from the Euler-Lotka equation

$$
1=\sum_{x=0}^{n} l_{x} m_{x} e^{-r x}
$$


where $l_{x}$ is the age-specific survivorship; $m_{x}$ is the age-specific fecundity (number of neonates per individual); and $x$ is the age at reproduction (in days).

\subsubsection{Gene expression experiment}

Simultaneously to the life history experiment we raised animals for the gene expression study; treatments consisted of the same three algae $S$. obliquus, N. limnetica, and Cryptomonas sp. Animals were raised in $1.5 \mathrm{~L}$ beakers each containing 20 individuals. Cultivation, frequency of transfer and amounts of food were as described for the life history experiment. After releasing their second clutch offspring daphnids were sampled using a "cylindrical sieve system" (Heckmann et al., 2007) and stored at $-80^{\circ} \mathrm{C}$ in $400 \mu \mathrm{L}$ RNAlater $B$ (Ambion) for subsequent RNA extraction.

\subsection{Gene expression analysis}

\subsubsection{RNA extraction and DNA synthesis}

Total RNA was extracted using the RNeasyMini kit with on-column DNase treatment (Qiagen) according to the manufacturer's instructions. RNA concentrations were determined using a NanoDrop ${ }^{\mathrm{TM}}$ spectrophotometer (NanoDrop Technologies). Agarose (1.5\%) gel electrophoresis was used to verify the quality of RNA. cDNA was synthesized from $4 \mu \mathrm{g}$ total RNA using the First Strand cDNA Synthesis Kit (Fermentas) following the manufacturer's instructions. Subsequently, cDNA was diluted 50 -fold to a concentration equivalent to $4 \mathrm{ng}$ total $\mathrm{RNA}_{\mu \mathrm{L}}{ }^{-1}$ and stored at $-20^{\circ} \mathrm{C}$

\subsubsection{Relative expression of $m R N A$}

Analysis comprised eight genes: Cox (cyclooxygenase), Gpx (glutathione peroxidase), Clect (C-type lectin like), Ltb4dh (leukotriene $\mathrm{B}_{4}$ 12-hydroxydehydrogenase), DmagVtg1 (vitellogenin 1), Jhe (juvenile hormone esterase), Lip (triacylglycerol lipase), Fabp3 (fatty acid binding protein 3). Primer sequences were taken from Heckmann et al. (2008a) (additional data files 3, 8); primers were synthesized by biomers.net (Ulm, Germany). Real-time quantitative polymerase chain reactions (qPCR) were conducted on a Stratagene MX3005P (AH Diagnostics) using Stratagene Brilliant ${ }^{\circledR}$ II SYBR ${ }^{\circledR}$ Green qPCR Mastermix (AH Diagnostics). Each reaction was run in duplicate and contained $5 \mu \mathrm{L}$ of cDNA template (equivalent to $20 \mathrm{ng}$ total RNA) along with $900 \mathrm{nM}$ primers in a final volume of $15 \mu \mathrm{L}$. The amplification was performed under the following conditions: $95^{\circ} \mathrm{C}$ for $10 \mathrm{~min}$ to activate the DNA polymerase, then 40 cycles of $95^{\circ} \mathrm{C}$ for $10 \mathrm{~s}$ and $60{ }^{\circ} \mathrm{C}$ for $60 \mathrm{~s}$. Melting curves were visually inspected to verify a single amplification product with no primer-dimers. No inconsistencies were detected in the present data set.

\subsection{Data analysis and statistics}

Raw qPCR data were analyzed using Data Analysis for Real-Time PCR (DART-PCR) (Peirson et al., 2003). The calculated reaction efficiencies verified the expected amplification of around 2 -fold for all genes. The few outliers detected were removed. The associated melting curves were inspected to verify the presence of a single and specific amplicon. The resulting data set was normalized by NORMA-Gene (Heckmann et al., 2011). Differences in relative normalized expression of the target genes among treatments were assessed using one-way analysis of variance (ANOVA) if assumptions of normality and homogeneity of variances were met. If assumptions were violated, Kruskal-Wallis one-way ANOVA on ranks was performed. For illustration, gene expressions of animals fed N. limnetica or Cryptomonas sp. were calibrated to those of animals fed $S$. obliquus, i.e. the relative expression for the $S$. obliquus treatment was fixed to 1 . All statistics were carried out using Sigmaplot (v 12.0, Systat Software) or Statistica (v 6.0, StatSoft).

\section{Results}

\subsection{Elemental stoichiometry and lipid composition of food organism}

The elemental composition of the food organisms was characterized by high contents of nitrogen and phosphorus and low C:N and C:P ratios (means \pm s.d.; S. obliquus: C:N 17.2 $\pm 0.6, C: P$ 201.3 \pm 9.7 ; N. limnetica: C:N 10.5 $\pm 3.6, C: P 129.5 \pm 5.1$; Cryptomonas sp.: C:N $5.4 \pm 0.1, \mathrm{C}: \mathrm{P} 145.6 \pm 8.7)$. A limitation of $D$. magna by $\mathrm{N}$ or $\mathrm{P}$ could therefore be excluded.

The three food organisms differed notably in their PUFA composition (Table 1). S. obliquus contained linoleic acid (LIN, 18:2n-6), $\gamma$-linolenic acid (GLA, 18:3n-6), $\alpha$-linolenic acid (ALA, 18:3n-3), and stearidonic acid (SDA, 18:4n-3), but no PUFAs with more than 18 carbon atoms were detected. $N$. limnetica contained LIN and the C20 PUFAs ARA and EPA, the latter in exceptionally high amounts. In Cryptomonas sp., we found LIN, ALA, SDA, EPA and docosahexaenoic acid (DHA, 22:6n-3). The amounts of total saturated fatty acids (SAFAs) and total monounsaturated fatty acids (MUFAs) were highest in N. limnetica; S. obliquus and Cryptomonas sp. were comparable concerning the total amount of SAFAs, but Cryptomonas sp. contained less MUFAs. The total PUFA content was generally higher than the SAFA or MUFA content in all three algae. Cryptomonas sp. contained the highest amounts of total PUFA followed by $N$. limnetica; both algae contained more PUFAs than S. obliquus. Only N. limnetica and Cryptomonas sp. contained C20 PUFAs, the amount in $N$. limnetica being almost four times that in Cryptomonas sp.

\subsection{Reproduction and population growth rates}

Cumulative numbers of viable offspring produced within the first three reproduction cycles and the estimated population growth rates increased significantly from animals fed $S$. obliquus to animals fed $N$. limnetica and again to those fed Cryptomonas sp. (Fig. 1a and b). The differences in cumulative offspring numbers caused by different algal diets were already apparent in the first clutch, where both $N$. limnetica and Cryptomonas sp. as diet for D. magna led to significantly larger clutch sizes compared to a $S$. obliquus diet, but they were most pronounced after three reproduction cycles (Fig. 1a).

\subsection{Expression of target genes}

The expression of all examined genes was influenced by the food treatments (Fig. 2), only two were not significantly affected (DmagVtg1, $\mathrm{p}=0.156 ;$ Ltb4dh, $\mathrm{p}=0.228$ ).

The most prominent changes in gene expressions were observed for Cox, Fabp3, and Clect. Cox was up-regulated $\sim 3$-fold in both the N. limnetica and Cryptomonas sp. treatment. Fabp3, in contrast, was down-regulated $\sim 4$-fold when $N$. limnetica or Cryptomonas sp. was used

Table 1

Fatty acid composition of S. obliquus, N. limnetica and Cryptomonas sp. Data are means of three replicates \pm s.d. expressed in $\mu \mathrm{g} \mathrm{m} \mathrm{C}^{-1}$ (n.d. = not detectable).

\begin{tabular}{llll}
\hline & S. obliquus & N. limnetica & Cryptomonas sp. \\
\hline $18: 2 \mathrm{n}-6$ (LIN) & $39.1 \pm 1.3$ & $6.0 \pm 0.8$ & $23.7 \pm 0.7$ \\
$18: 3 n-6$ (GLA) & $3.0 \pm 0.1$ & n.d. & $n . d$. \\
$18: 3 n-3$ (ALA) & $60.1 \pm 0.9$ & n.d. & $68.5 \pm 2.1$ \\
$18: 4 n-3$ (STA) & $8.2 \pm 0.1$ & n.d. & $21.3 \pm 0.6$ \\
$20: 4 n-6$ (ARA) & n.d. & $24.1 \pm 2.5$ & $n . d$. \\
$20: 5 n-3$ (EPA) & n.d. & $124.4 \pm 12.4$ & $36.4 \pm 0.7$ \\
$22: 6 n-3$ (DHA) & n.d. & $n . d$. & $4.4 \pm 0.0$ \\
Total SAFAs & $46.6 \pm 2.4$ & $73.6 \pm 6.0$ & $42.2 \pm 0.3$ \\
Total MUFAs & $82.9 \pm 7.9$ & $125.7 \pm 2.6$ & $3.4 \pm 0.1$ \\
Total PUFAs & $110.4 \pm 2.2$ & $145.4 \pm 1.7$ & $154.4 \pm 2.8$ \\
Total C20 PUFAs & $n . d$. & $139.8 \pm 1.6$ & $36.4 \pm 0.7$ \\
Total FA & $239.8 \pm 9.1$ & $344.7 \pm 6.0$ & $200.0 \pm 2.4$ \\
\hline
\end{tabular}



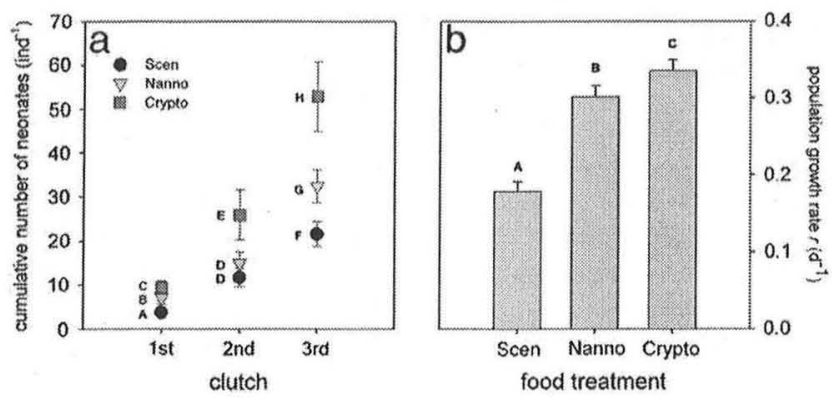

Fig. 1. Cumulative numbers of viable neonates produced per individual (a) and estimated population growth rates (b) of D. magna raised on S. obliquus (Scen), N. limnetica (Nanno) or Cryptomonas sp. (Crypto). Data are means of 16 replicates + s.e. Symbols or bars labelled with the same letters are not significantly different (Tukey's HSD test, $\mathrm{p}<0.05$ following ANOVA). Comparisons were carried out within each clutch.

as food. Similarly, Clect was down-regulated $\sim 3$ - and 4-fold with N. limnetica and Cryptomonas sp. as food, respectively.

Changes in the expression of Lip, Gpx, and Jhe were less distinct. While Lip was clearly up-regulated in the $N$. limnetica treatment $(\sim 3.5$-fold $)$, this was less pronounced in the Cryptomonas sp. treatment ( 2-fold). The expression of Gpx did not differ in animals fed S. obliquus and N. limnetica, but was slightly reduced in animals fed
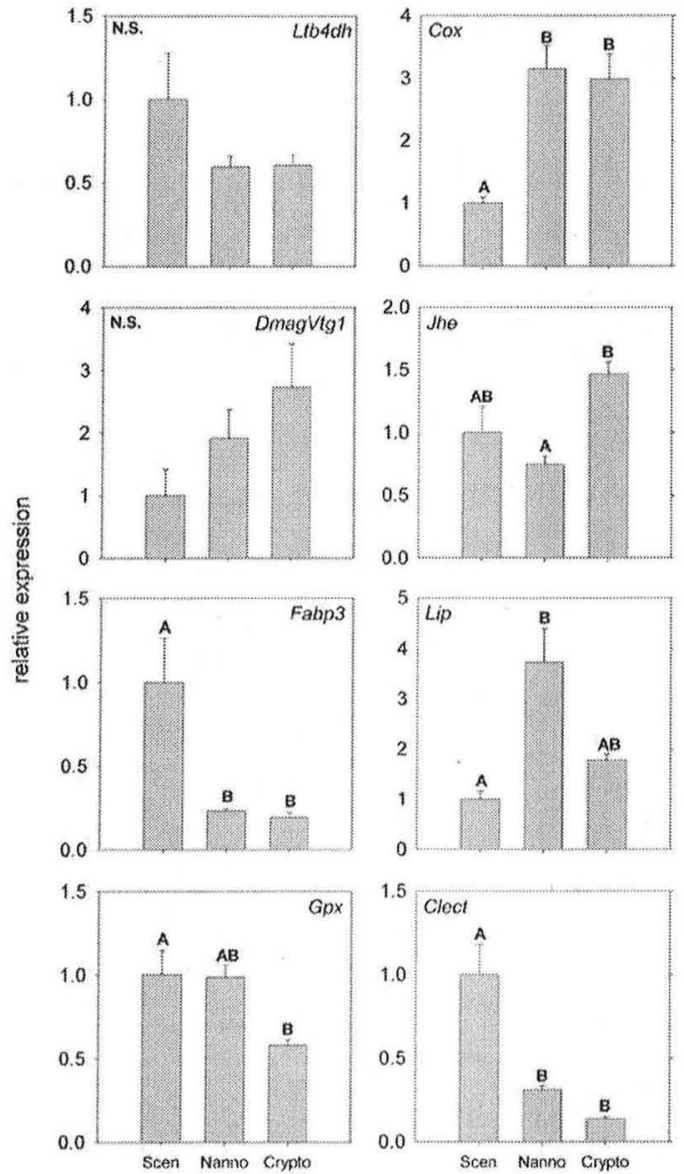

food treatment

Fig. 2. Relative expression of eight selected genes of D. magna raised on S. obliquus (Scen), N. limnetica (Nanno) or Cryptomonas sp. (Crypto). For graphic presentation, expression levels are related to gene expression observed on a $S$. obliquus diet (=calibrator). Shown are mean relative expressions $(\mathrm{N}=3 \pm$ s.e.). Bars labelled with the same letters are not significantly different (Tukey's HSD test, $\mathrm{p}<0.05$ following ANOVA or KruskalWallis). N.S. = not significant.
Cryptomonas sp. ( 2-fold). Jhe-expression differed in animals feeding on N. limnetica and Cryptomonas sp., but both treatments did not differ significantly from the expression level obtained with S. obliquus as food.

Although not significant, the expression levels of Ltb4dh as well as DmagVtg1 were clearly affected by the food sources. $L t b 4 d h$ expression was almost halved in animals feeding on $N$. limnetica or Cryptomonas sp. whereas DmagVtg1 displayed the opposite trend, i.e. its expression was up-regulated 2- and 3-fold in animals fed $\mathrm{N}$. limnetica and Cryptomonas sp., respectively, compared to animals fed S. obliquus.

\section{Discussion}

As a first approach to link biochemical nutrient availabilities to gene expression, we raised Daphnia magna on food sources differing in their PUFA composition and assessed associated changes in the expression of selected candidate genes. We found all of these candidate genes to be responsive to the different food sources and propose that the observed effects on gene expression can be mainly attributed to differences in the availability of dietary fatty acids. Differences in fatty acid profiles among the three algae used as food for $D$. magna are substantial and are expected to be responsible for the superior food quality of $N$. limnetica and Cryptomonas sp. as was observed in the concomitantly performed life history experiment where both algae rich in PUFAs $(>18 \mathrm{C}$ ) yielded higher offspring numbers and population growth rates than S. obliquus. Although the elemental nutrient ratios ( $\mathrm{C}: \mathrm{P}$ and $\mathrm{C}: \mathrm{N}$ ) found in the three algae differed slightly, both $\mathrm{N}$ and $\mathrm{P}$ are present in a supply far from what is thought to be limiting for Daphnia (Sterner and Elser, 2002).

In contrast to S. obliquus, N. limnetica and Cryptomonas sp. contain C20 PUFAs which in Daphnia can serve as precursors for eicosanoid biosynthesis. Only $N$. limnetica contains ARA, the main substrate for this biosynthesis. In addition, the amounts of EPA found in this alga are exceptionally high. Genes examined here included representatives of different pathways possibly influenced by changing dietary PUFA availabilities, as indicated by their response to the eicosanoid biosynthesis inhibitor ibuprofen (Heckmann et al., 2008a). Hayashi et al. (2008) reported that reproduction of daphnids is impaired upon exposure to ibuprofen, which is in line with the finding that dietary C20 PUFAs, i.e. eicosanoid precursors, increase the reproductive success of daphnids (Martin-Creuzburg et al., 2010). Vitellogenin, a lipoprotein and precursor of the major egg yolk protein vitellin, is indispensable for oogenesis and thus reproduction (Bownes, 1986). Therefore, one would expect that a higher egg number is accompanied by an increase in the expression of DmagVtg1. Although the increase in transcription of DmagVtg1 from S. obliquus to N. limnetica and further to Cryptomonas sp. was not significant due to the comparatively large within-treatment variance, this increase nicely reflects the increasing reproductive output of animals feeding on these diets.

Besides DmagVtg1, Jhe expression should be considered when looking at oogenesis as the encoded esterase cleaves the active juvenile hormone thereby rendering it inactive and as a consequence releases vitellogenin from the suppressive influence of juvenile hormone (Tokishita et al., 2006). If an increase in DmagVtg1 expression would require a synchronous increase in JHE, we would expect to find a similar expression pattern for both genes. However, we were examining mature, reproducing animals and as increased levels of juvenile hormone may be disruptive of reproduction we would not expect to find any differences in Jhe expression among the three algae. Methyl farnesoate, the crustacean juvenile hormone, has been shown to induce male production in D. magna (Olmstead and Leblanc, 2002) and hence should be suppressed under favourable standard experimental conditions. In this study, all experimental animals reproduced parthenogenetically, i.e. no males were observed, and the expression of Jhe in animals raised on $N$. limnetica or Cryptomonas $\mathrm{sp}$. was not significantly different from that in animals raised on S. obliquus. Hence, one might 
argue against a connection between vitellogenesis and juvenile hormone synthesis and, therefore, against a role for PUFAs in juvenile hormone signalling.

Lip codes for a triacylglycerol lipase and hence is directly involved in lipid metabolism, particularly in the glycerolipid metabolism (Prentki and Madiraju, 2008). Lip was up-regulated in animals feeding on $N$. limnetica, and also to a smaller extend in animals feeding on Cryptomonas sp. The strong increase in Lip expression in animals feeding on $N$. limnetica is most likely a general response to the high SAFA and/or total fatty acid content of $N$. limnetica rather than a specific, PUFA-related response. LIP presumably is a secreted protein as suggested by the presence of a predicted signal sequence; hence, the observed increase in Lip expression might indicate an increased lipid assimilation process, in which available fatty acids in the diet are released from triacylglycerides in the gut through the activity of lipases, absorbed through the epithelium, and subsequently processed and stored in lipid droplets (Mu and Hoy, 2004; Lass et al., 2011).

Lectins act as recognition molecules within the immune system and are, through their ability to bind glucoproteins and glycolipids and to mediate e.g. endocytosis, important modules of pathogen defence (Kilpatrick, 2002). Clect expression was down-regulated in both $N$. limnetica and Cryptomonas sp. consuming animals. This pattern may represent a trade-off situation between immune response and reproductive output, i.e. an increased allocation of resources toward reproduction in animals fed $N$. limnetica or Cryptomonas sp. might lead to a reduced investment in components of the immune system. Indications for a trade-off of immunity for other fitness parameters have been described in many organisms (Zuk and Stoehr, 2002; Allen and Little, 2011). Rono et al. (2010) observed interference of vitellogenin with the immune response to an invading pathogen in Anopheles gambiae.

Another group of genes can be associated directly with eicosanoid metabolism as they are coding for the required enzymes: COX, one of the central enzymes in the biosynthesis of eicosanoids and LTB4DH, an enzyme responsible for rendering some eicosanoids inactive (Stanley, 2000); GPX provides protection against toxic oxygen derivatives and is able to reduce lipid hydroperoxides formed during eicosanoid synthesis (Schoene, 1985; Arthur, 2000).

As key enzyme within the eicosanoid biosynthesis pathway, we expected the Cox gene to be highly responsive to changes in substrate availability, i.e. the availability of the three relevant C20 PUFAs. In both the $N$. limnetica and Cryptomonas sp. treatment, where these C20 precursors are present, gene expression levels were clearly elevated compared to $S$. obliquus. This might reflect an induction of the Cox gene by the presence of the substrate (ARA or EPA) of the encoded enzyme and would thus indicate a higher rate of eicosanoid synthesis. Supporting this possibility is the concomitantly slightly lowered $L t b 4 d h$ expression in animals fed $N$. limnetica or Cryptomonas sp., as the enzyme encoded by this gene is, at least in vertebrates, responsible for the inactivation of eicosanoids. Moreover, leukotriene $\mathrm{B}_{4}$ $\left(\mathrm{LTB}_{4}\right)$ has been shown to play a role in yolk formation during oogenesis in insects (Medeiros et al., 2004). As Ltb4dh expression is downregulated in animals raised on a $\mathrm{N}$. limnetica or Cryptomonas $\mathrm{sp}$. diet, there should be more $\mathrm{LTB}_{4}$ present, which in turn would (according to Medeiros et al.) support yolk formation. Together with the increased expression of the vitellogenin gene DmagVtg1 in animals fed the C20 PUFA-rich diets in our experiment, this argues for a similar role of $\mathrm{LTB}_{4}$ in insect and Daphnia reproduction.

Glutathione peroxidases reduce the hydroperoxides formed by ARA metabolism during eicosanoid synthesis. Although eicosanoids exert crucial biological activities their peroxide nature can also cause severe damage to membranes. Hence, one would expect a more pronounced expression of $G p x$ in animals that are able to extensively synthesize eicosanoids, i.e. in our case, in animals feeding on the C20 PUFA-rich algae $N$. limnetica or Cryptomonas sp. Yet, we did not find the expected up-regulation of $G p x$ expression. While Gpx expression levels did not differ between animals fed S. obliquus and $N$. limnetica, they were slightly lower in the Cryptomonas sp. treatment. Whether or not animals feeding on Cryptomonas sp. are in fact exposed to lower hydroperoxide-mediated stress than animals feeding on $S$. obliquus and $N$. limnetica remains unclear and has to be re-assessed in future studies.

Another gene closely related to the eicosanoid pathway is Fabp3. Fatty acid binding proteins (FABPs) are involved in signalling processes of vertebrates and invertebrates as intracellular carrier proteins for fatty acids, eicosanoids and other lipophilic substances (Zimmerman and Veerkamp, 2002; Esteves and Ehrlich, 2006). Possible destinations for both free fatty acids and eicosanoids bound to FABPs can be peroxisome proliferator-activated receptors (PPARs). These nuclear receptors dimerize upon ligand binding (i.e. activation) with the retinoid $X$ receptor ( $R X R)$ and the resulting complex consequently binds to the promoter region of target genes (Berger and Moller, 2002). In our study, the expression pattern of Fabp3 in animals fed the different food sources was similar to the expression pattern of Ltb4dh, but reversed to that of Cox, which implies a connection to eicosanoid signalling. However, the reason for the significant down-regulation of Fabp3 in animals feeding on the C20 PUFA-containing algae $N$. limnetica and Cryptomonas sp. requires further investigation, in which receptors involved in the above described signaling cascade, e.g. PPAR or RXR, are considered. Also, as for all genes examined, it remains to be tested if the observed changes in gene expression are reflected in changes in the functional protein.

Eicosanoids play an important role in reproduction and immunity of many invertebrates (Stanley, 2000). So far there are only few hints as to their significance in Daphnia. Hayashi et al. (2008) used the eicosanoid synthesis inhibitor ibuprofen to assess its toxicity for $D$. magna and found strong concentration dependent effects on reproduction. Here, we demonstrated effects of food quality on reproduction in a life history experiment and hypothesized that PUFAs, as one of the major factors distinguishing the employed algae, exert their influence on offspring production in part through eicosanoid actions. As the relevance of PUFAs for Daphnia reproduction is well-established (e.g. Martin-Creuzburg et al., 2010) and the necessary enzymatic machinery for eicosanoid synthesis exists in Daphnia (Heckmann et al., 2008b; Colbourne et al., 2011), we are directing future studies along this line of thought. To elucidate the role of PUFAs in eicosanoid production further studies are required in which the dietary PUFA supply is experimentally modified by specific PUFA supplementation. It also has to be considered that the activity of COX is potentially influenced by other dietary substances, such as carotenoids (Reddy et al., 2005). With this approach we hope to get further insights in how PUFAs act in Daphnia physiology and to establish a link between dietary PUFA availability and eicosanoid synthesis.

\section{Acknowledgment}

We thank Lars-Henrik Heckmann for advice, discussion and valuable comments on the manuscript. This study was supported financially by the German Research Foundation (DFG, MA 5005/1-1).

\section{References}

Allen, D.E., Little, T.J., 2011. Identifying energy constraints to parasite resistance. J. Evol. Biol. 24, 224-229.

Arthur, J.R., 2000. The glutathione peroxidases. Cell. Mol. Life Sci. 57, 1825-1835.

Berger, J., Moller, D.E., 2002. The mechanisms of action of PPARs. Annu. Rev. Med. 53 409-435.

Bownes, M., 1986. Expression of the genes coding for vitellogenin (yolk protein). Annu. Rev. Entomol. 31, 507-531.

Colbourne, J.K., Pfrender, M.E., Gilbert, D., Thomas, W.K., Tucker, A., Oakley, T.H., Tokishita, S., Aerts, A., Arnold, G.J., Basu, M.K., Bauer, D.J., Caceres, C.E., Carmel, L. Casola, C., Choi, J.H., Detter, J.C., Dong, Q.F., Dusheyko, S., Eads, B.D., Frohlich, T. Geiler-Samerotte, K.A., Gerlach, D., Hatcher, P., Jogdeo, S., Krijgsveld, J., Kriventseva, E.V., Kultz, D., Laforsch, C., Lindquist, E., Lopez, J., Manak, J.R., Muller, J., Pangilinan, J., Patwardhan, R.P., Pitluck, S., Pritham, E.J., Rechtsteiner, A., Rho, 
M., Rogozin, I.B., Sakarya, O., Salamov, A., Schaack, S., Shapiro, H., Shiga, Y., Skalitzky, C., Smith, Z., Souvorov, A., Sung, W., Tang, Z.J., Tsuchiya, D., Tu, H., Vos, H., Wang, M., Wolf, Y.I., Yamagata, H., Yamada, T., Ye, Y.Z., Shaw, J.R., Andrews, J. Crease, T.J., Tang, H.X., Lucas, S.M., Robertson, H.M., Bork, P., Koonin, E.V. Zdobnov, E.M., Grigoriev, I.V., Lynch, M., Boore, J.L., 2011. The ecoresponsive genome of Daphnia pulex. Science 331, 555-561.

Ebert, D., 2005. Ecology, Epidemiology, and Evolution of Parasitism in Daphnia. National Library of Medicine (US), National Center for Biotechnology Information, Bethesd (MD).

Ebert, D., 2011. A genome for the environment. Science 331, 539-540.

Esteves, A., Ehrlich, R., 2006. Invertebrate intracellular fatty acid binding proteins. Comp. Biochem. Physiol. C 142, 262-274.

Greenberg, A.E., Trussell, R.R., Clesceri, L.S., 1985. Standard methods for the examination of water and wastewater. American Public Health Association, Washington, DC

Guillard, R.R.L., 1975. Cultures of phytoplankton for feeding of marine invertebrates. In: Smith, W.L., Chanley, M.H. (Eds.), Culture of Marine Invertebrate Animals. Plenum.

Guschina, I.A., Harwood, J.L., 2006. Mechanisms of temperature adaptation in poikilotherms. FEBS Lett. 580, 5477-5483.

Hayashi, Y., Heckmann, L.H., Callaghan, A., Sibly, R.M., 2008. Reproduction recovery of the crustacean Daphnia magna after chronic exposure to ibuprofen. Ecotoxicology 17, 246-251.

Heckmann, L.H., Bouetard, A., Hill, C.J., Sibly, R.M., Callaghan, A., 2007. A simple and rapid method for preserving RNA of aquatic invertebrates for ecotoxicogenomics. Ecotoxicology 16, 445-447.

Heckmann, L.H., Sibly, R.M., Connon, R., Hooper, H.L., Hutchinson, T.H., Maund, S.J., Hill, C.J., Bouetard, A., Callaghan, A., 2008a. Systems biology meets stress ecology: linking molecular and organismal stress responses in Daphnia magna. Genome Biol. 9, R40.

Heckmann, L.H., Sibly, R.M., Timmermans, M., Callaghan, A., 2008b. Outlining eicosanoid biosynthesis in the crustacean Daphnia. Front. Zool. 5, 11.

Heckmann, L.H., Sorensen, P.B., Krogh, P.H., Sorensen, J.G., 2011. NORMA-Gene: a simple and robust method for qPCR normalization based on target gene data. BMC Bioinforma. 12, 7

Kilpatrick, D.C., 2002. Animal lectins: a historical introduction and overview. BBA Gen Subj. 1572, 187-197.

Klüttgen, B., Dülmer, U., Engels, M., Ratte, H.T., 1994. ADaM, an artificial freshwater for the culture of zooplankton. Water Res. 28, 743-746,

Koch, U., Martin-Creuzburg, D., Grossart, H.-P., Straile, D., 2011. Single dietary amino acids control resting egg production and affect population growth of a key freshwater herbivore. Oecologia 167, 981-989.

Lampert, W., 1977. Studies on the carbon balance of Daphnia pulex as related to environmental conditions part 1: methodological problems of the use of ${ }^{14} \mathrm{C}$ for the measurement of carbon assimilation. Arch. Hydrobiol. Suppl. 48, 287-309.

Lampert, W., 2011. Daphnia: Development of a Model Organism in Ecology and Evolution. International Ecology Institute, Oldendorf/Luhe.

Lass, A., Zimmermann, R., Oberer, M., Zechner, R., 2011. Lipolysis - a highly regulated multi-enzyme complex mediates the catabolism of cellular fat stores. Prog. Lipid Res. 50, 14-27.

Martin-Creuzburg, D., von Elert, E., Hoffmann, K.H., 2008. Nutritional constraints at the cyanobacteria-Daphnia magna interface: the role of sterols. Limnol. Oceanogr. 53. 456-468.

Martin-Creuzburg, D., Sperfeld, E., Wacker, A., 2009. Colimitation of a freshwater herbivore by sterols and polyunsaturated fatty acids. Proc. R. Soc. B Biol. Sci. 276 1805-1814.

Martin-Creuzburg, D., Wacker, A., Basen, T., 2010. Interactions between limiting nutrients: consequences for somatic and population growth of Daphnia magna. Limnol. Oceanogr $55,2597-2607$

Martin-Creuzburg, D., Beck, B., Freese, H.M., 2011. Food quality of heterotrophic bacteria for Daphnia magna: evidence for a limitation by sterols. FEMS Microbiol. Ecol. 76 , 592-601.
Martin-Creuzburg, D., Wacker, A., Ziese, C., Kainz, M., 2012. Dietary lipid quality affects temperature-mediated reaction norms of a freshwater key herbivore. Oecologia $168,901-912$.

Masclaux, H., Bec, A., Kainz, M.J., Desvilettes, C., Jouve, L., Bourdier, G., 2009. Combined effects of food quality and temperature on somatic growth and reproduction of two freshwater cladocerans. Limnol. Oceanogr. 54, 1323-1332.

Medeiros, M.N., Mendonca, L.H., Hunter, A.L., Paiva-Silva, G.O., Mello, F.G., Henze, I.P. Masuda, H., Maya-Monteiro, C.M., Machado, E.A., 2004. The role of lipoxygenase products on the endocytosis of yolk proteins in insects: participation of cAMP. Arch. Insect Biochem. 55, 178-187.

Mouritsen, O.G., Zuckermann, M.J., 2004. What's so special about cholesterol? Lipids $39,1101-1113$.

Mu, H.L., Hoy, C.E., 2004. The digestion of dietary triacylglycerols. Prog. Lipid Res. 43. 105-133.

Müller-Navarra, D.C., 1995. Evidence that a highly unsaturated fatty acid limits Daphni growth in nature. Arch. Hydrobiol. 132, 297-307.

Müller-Navarra, D.C., Brett, M.T. Liston, A.M. Goldman, C.R., 2000. A highly unsaturated fatty acid predicts carbon transfer between primary producers and consumers. Nature 403, 74-77.

Mykles, D.L., 2011. Ecdysteroid metabolism in crustaceans. J. Steroid Biochem. 127 196-203.

Olmstead, A.W., Leblanc, G.A., 2002. Juvenoid hormone methyl farnesoate is a sex determinant in the crustacean Daphnia magna. J. Exp. Zool. 293, 736-739.

Peirson, S.N., Butler, J.N., Foster, R.G., 2003. Experimental validation of novel and conventional approaches to quantitative real-time PCR data analysis. Nucleic Acids Res. 31, 7.

Pietrzak, B., Grzesiuk, M., Bednarska, A., 2010. Food quantity shapes life history and survival strategies in Daphnia magna (Cladocera). Hydrobiologia 643, 51-54.

Prentki, M., Madiraju, S.R.M., 2008. Glycerolipid metabolism and signaling in health and disease. Endocr. Rev. 29, 647-676.

Reddy, M.K., Alexander-Lindo, R.L., Nair, M.G., 2005. Relative inhibition of lipid peroxidation, cyclooxygenase enzymes, and human tumor cell proliferation by natural food colors. J. Agric. Food Chem. 53, 9268-9273.

Rono, M.K., Whitten, M.M.A., Oulad-Abdelghani, M., Levashina, E.A., Marois, E., 2010 The major yolk protein vitellogenin interferes with the anti-Plasmodium response in the malaria mosquito Anopheles gambiae. PloS Biol. 8, e1000434.

Schoene, N.W., 1985. Selenium-dependent glutathione peroxidase and eicosanoid production. In: Lands, E.M. (Ed.), Biochemistry of Arachidonic Acid Metabolism. Martinus Nijhoff Publishing, Boston.

Stanley, D.W., 2000. Eicosanoids in Invertebrate Signal Transduction Systems. Princeton University Press, Princeton, NJ.

Sterner, R.W., Elser, J.J., 2002. Ecological Stoichiometry: The Biology of Elements from Molecules to the Biosphere. Princeton University Press, Princeton, NJ.

Tokishita, S., Kato, Y., Kobayashi, T., Nakamura, S., Ohta, T., Yamagata, H., 2006. Organization and repression by juvenile hormone of a vitellogenin gene cluster in the crustacean, Daphnia magna. Biochem. Biophys. Res. Commun. 345, 362-370.

van Meer, G., Voelker, D.R., Feigenson, G.W., 2008. Membrane lipids: where they are and how they behave. Nat. Rev. Mol. Cell Biol. 9, 112-124.

von Elert, E., 2002. Determination of limiting polyunsaturated fatty acids in Daphnia galeata using a new method to enrich food algae with single fatty acids. Limnol. Oceanogr. 47, 1764-1773.

Wacker, A., Martin-Creuzburg, D., 2007. Allocation of essential lipids in Daphnia magna during exposure to poor food quality. Funct. Ecol. 21, 738-747.

Wacker, A., Von Elert, E., 2001. Polyunsaturated fatty acids: evidence for nonsubstitutable biochemical resources in Daphnia galeata. Ecology 82, 2507-2520.

Zimmerman, A.W., Veerkamp, J.H., 2002. New insights into the structure and function of fatty acid-binding proteins. Cell. Mol. Life Sci. 59, 1096-1116.

Zuk, M., Stoehr, A.M., 2002. Immune defense and host life history. Am. Nat. 160, 9-22. 